\section{DNA vaccines-back in the saddle again?}

A promising new horse vaccine may reignite enthusiasm for DNA vaccine technology in designing prophylactics against infectious disease. Kendall Powell reports.

Quietly weaving its way through the final stages of registration with the US Department of Agriculture, a horse vaccine for West Nile virus may soon make history as the first approved DNA vaccine. The vaccine, produced by Fort Dodge Animal Health, which is in Fort Dodge, Iowa, may not be an odds-on winner commercially because a conventional West Nile vaccine already exists for horses, but the product could usher in the next generation of protective and immunotherapeutic DNA vaccines. It could also serve as a trailblazer product for a technology that has often failed to repeat preclinical success in the clinic.

\section{Getting out of the gate}

When the concept of a DNA vaccine first popped onto the scene in the early $1990 \mathrm{~s}^{1,2}$, it seemed too simple, too easy and too bizarre to be true. Traditional vaccine development involves either passing a pathogenic virus through a different host species or cell type until it becomes attenuated or killing the virus in a manner that maintains its immunogenicity; both processes involve guesswork and many laborious years to develop.

In contrast, DNA vaccines rely on a ring of nucleic acid to encode an antigen that when taken up by the immune system, elicits a response strong enough to protect against infectious disease. Such plasmids can hold multiple genes for multiple antigens and recombinant DNA technology offers an easy way to mix-and-match antigens in new combinations. Over the years, two general approaches to DNA vaccines have emerged: one in which recombinant viral vectors were used to deliver DNA, the other in which the DNA vaccine was delivered as a naked plasmid. Although many scoffed at the notion that a DNA sequence could elicit immunity, within a few years experimental data showed that rodents injected with foreign genes expressed antigens, produced antibodies and achieved a protective, long-lasting immune response against infectious diseases, such as hepatitis B, herpes virus and $\mathrm{HIV}^{3}$. And the field enthusiastically took off.
The excitement waned, though, as products moved into primate studies and human safety trials. In the jump to primate immune systems, plasmids failed to make the grade, generating little-to-no response. Because safety trials require tests for immune reaction to the DNA injection, it became obvious that there was no immune reaction to the antigens encoded by the plasmid, either. (For this same reason, phase 1 trials in the field are essentially phase $1 / 2$ because the immune reaction is an intermediate correlate for efficacy.) The letdown forced companies to reevaluate the technology.

"We have done a lot of work with adjuvants to increase immunogenicity and lower the dose," reports Vijay Samant, president and CEO of San Diego-based Vical, a leading company in naked DNA vaccines. He also says that during the past decade, plasmids have been optimized for production and expression efficiency. "It's like Hong Kong real estate-we've done everything we can do on the plasmid."

Great strides have also been made in delivering plasmid DNA vaccines to the correct types of cells-antigen-presenting dendritic cells found within skin and muscle tissue-and into their nuclei for effective expression (see Box 1). And finally, a 'prime-boost' strategy has emerged that may take advantage of the best of both naked DNA plasmid and recombinant viral vector vaccines. Although plasmid DNA does not always evoke a strong immune response, it can be used to 'prime' the immune system to recognize the same antigens more effectively when they are delivered later by a viral vector booster. Although the priming mechanism is unclear, it has now been demonstrated to increase vector and protein-based vaccine effects in several systems.

\section{Jockeying for position}

The improvements in plasmid delivery, antigen expression and adjuvant or priming strategies have investors and researchers hopeful once again, particularly about vaccines that protect against infectious diseases. If it comes to pass, approval of Fort Dodge's West Nile virus horse vaccine will be a milestone. Although Fort Dodge already manufactures a

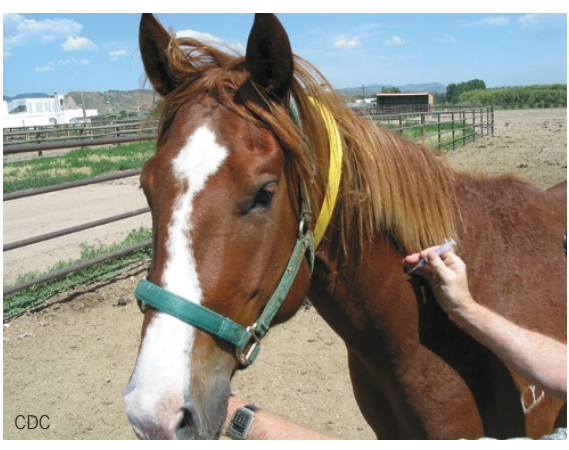

An equine vaccine against West Nile virus is in its final stages of registration.

marketed vaccine for West Nile virus, Terry $\mathrm{Ng}$, associate director for vaccine development, explains that the company wants to build a platform of DNA vaccine technology for other veterinary applications. $\mathrm{Ng}$ notes that Fort Dodge has added its proprietary adjuvant to the DNA plasmid that codes for the West Nile virus outer coat proteins.

"The technology is making a comeback," say Gary Nabel, director of the US National Institutes of Health (NIH) Vaccine Research Center (VRC) where several DNA vaccines are in or entering phase 1 clinical trials, including a human West Nile virus vaccine with the same gene insert as the equine version. "It would be a wonderful thing for the field if the vaccine is approved for veterinary use; it would be a proof-of-concept of the technology." But he cautions against unbridled enthusiasm just yet, noting important differences between the approval processes at the USDA and FDA and between horse and human immune responses.

However, the fact that a naked DNA vaccine was able to protect a large animal from West Nile virus suggests there are surprises still in store for the field. Jeff Chang, who developed the basic plasmid backbone used in both the Fort Dodge and VRC trials, explains how flaviviruses-the genus that West Nile virus belongs to - might be particularly good targets for DNA vaccines. Flaviviruses, which include Dengue virus and Japanese encephalitis virus, naturally produce empty particles lacking virus RNA (virus-like particles; VLPs) from infected cells. When coat proteins are put onto a plasmid, VLPs are formed and secreted by cells expressing the plasmid; these secreted and assembled VLPs give a much greater immune response than a surface protein or even a secreted protein by itself.

\section{A thoroughbred platform}

The ability to manipulate DNA vaccine antigens by recombinant means may also make them particularly suited to tackle some of the 


\section{Box 1 IP that delivers}

Since DNA vaccines represent a fundamental technology that any sparsely equipped molecular biology laboratory could produce, most intellectual property (IP) rights in this arena involve the delivery method used to get plasmids into muscle, skin or other cells. Early on, Vical recognized the potential of the 1990 finding that mouse muscle cells injected with DNA would express those gene sequences ${ }^{1}$ and filed for a broad patent on delivering naked DNA plasmids. That patent was granted in 1996, ensuring Vical's leadership and licensing power in the field.

But because DNA uptake delivered by intramuscular injection is passive and inefficient, others pursued technologies to speed DNA uptake or deliver DNA directly to cells' nuclei where expression occurs. British firm PowderMed, in Oxford, spun out from Chiron Corporation in May, kept the rights to its PowderJect gene-gun technology, which shoots DNA-carrying gold particles into skin cell nuclei at near-supersonic speed. The technology is being used in two of PowderMed's in-house chronic viral treatment programs (hepatitis $B$ and human papilloma virus) and in collaborations with Chiron (herpes simplex virus) and GlaxoSmithKline (HIV). Copernicus Therapeutics, which is in Cleveland, Ohio, has exclusive worldwide rights to a similar technology that compacts DNA into a particle small enough to enter nuclear pores. Their complexes, used in both vaccine and gene therapy applications, can be modified to target particular cell types and to raise or lower expression levels.

Electroporation, in which a brief electrical charge causes pores to open on a cell's outer membrane, has proved promising in animal trials for delivering DNA plasmids. On May 24, Merck, in Whitehouse Station, New Jersey, licensed the technology from Genetronics Biomedical in San Diego for use with two of its DNA vaccine antigens in return for an undisclosed upfront payment, milestones and future product royalties.

most vexing of infectious diseases that have resisted traditional drug treatment or conventional vaccination. In particular, the ability to design plasmids with multiple genes encoding multiple proteins offers a way to mix-andmatch antigens in new combinations. Such multiple antigen vaccines could prove useful against viruses, such as HIV, which have a tendency to mutate rapidly, as well as against trypanosomes, which express different antigens at different stages during their life cycle.
DNA vaccines make it theoretically possible to develop a single vaccine to protect against the five clades of HIV found worldwide or the four serotypes of Dengue virus. Antigens can also be fused to other genes, such as signal sequences from bacterial proteins known to boost immune response to infectious disease or to immune system receptors for applications in cancer (see Box 2). Maxygen, which is in Redwood City, California, has developed a cross-protective Dengue vaccine using its 'molecular breeding' technology. "Doing recombination in the test tube," says Robert Whalen, director of infectious disease research at Maxygen, "is a powerful way to put evolution on fast-forward."

In the case of the Dengue vaccine, the genes for envelope proteins from the four subtypes were forced to recombine in vitro. The combinations coded for chimeric proteins, which were tested to find one that raised antibodies to all four types. Whalen says the approach is also being tested for creating novel HIV antigens by combining parent molecules that would hopefully make conserved protein residues more immunogenic.

The VRC, in collaboration with Vical, has completed a phase 1 trial of a 'global' HIV vaccine that carries the envelope protein from the three most widespread of the five clades found worldwide. Nabel wouldn't reveal the unpublished results just yet, but says, "the bottom line is that we have reason to be optimistic that it will be possible to induce immune responses in humans with DNA." However, the VRC also plans a phase 2 trial for late 2004 of the DNA vaccine in combination with an adenoviral vector booster carrying the same genes. Other major programs are also putting more confidence into similar prime-boost strategies, signaling a critical turning point for the field.

\section{Rounding the bend}

The trend toward testing DNA vaccines as part of a combination trial can be seen in both the large vaccine producers like Merck, in Whitehouse Station, New Jersey, Aventis Pasteur, in Strasbourg, France, and Wyeth, in Madison, New Jersey, (see Table 1) as well as in smaller research companies. On March 23,

\section{Box 2 DNA vaccines for cancer}

In addition to being used to fight infectious disease, DNA vaccines are also being developed to augment cancer immunotherapy. For example, Vical has developed Allovectin-7, a DNA plasmid/lipid complex encoding HLA-B7 and $\beta 2$ microglobulin (which together form major histocompatibility complex I (MHC-I) antigen) for injection into melanoma tumors. According to Vical CEO Vijay Samant, introduction of MHC-I into the tumor activates the patient's immune system so that it can recognize self-tumor cells. The systemic effect creates tumor cell-killing T cells that can travel to other noninjected tumors as well. The vaccine is moving into phase 3 trials later this year and Vical has a Special Protocol Assessment filed with the US Food and Drug Administration for fast-track approval should certain trial end points be met.

Freda Stevenson, an immunologist at the University of Southampton in Britain, has designed another strategy for breaking tolerance in her DNA vaccine for lymphoma. The plasmid fuses a short gene sequence (fragment C) from tetanus toxin to the tumor antigen sequence of interest as a way to get the immune system to pay attention to the antigen, opening a "backdoor to generating tumor immunity," she says. Stevenson explains that DNA vaccines allow more specificity than traditional killed tumor cell vaccines. "You can measure the immune response because you know exactly what [antigen] is going in. But how can you measure a response to mashed up cells where you don't know the antigens?"

According to Stevenson, DNA is also much cheaper and more stable to produce under the FDA's good manufacturing practice standards than monoclonal antibody therapies or individualized tumor cell vaccines. LipoNova is a biotech company in Hannover, Germany, with a renal cell carcinoma whole cell vaccine expected to be approved by the European Medicine Evaluation Agency this year. The company anticipates this first-of-its-kind cancer vaccine will cost $€ 18,000(\$ 22,000)$ per treatment, whereas several sources guess that DNA vaccines will eventually cost between $\$ 1-\$ 50$ per dose. 
Los Angeles-based CytRx in collaboration with the University of Massachusetts Medical School (UMMS), which is in Worcester, and Kensington, Maryland-based Advanced BioScience Laboratories announced the initiation of a phase 1 trial for their HIV vaccine formulation, which includes a DNA prime component, followed by a recombinant protein booster.

Steve Kriegsman, CEO of CytRx, notes that investor confidence in DNA technology runs high - the company's stock rose $84 \%$ the day after announcing the licensing agreement with UMMS in May 2003 and the NIH has awarded a $\$ 16$ million grant for the clinical trial. In the DNA vaccine field, early research through phase 1 clinical trials tend to be heavily funded by grants from the US Centers for Disease Control, NIH or private charities.

Shan $\mathrm{Lu}$, a vaccinologist at UMMS, developed the plasmid component, which contains the envelope proteins of all five HIV clades plus a gag protein. He stresses that DNA plasmids allow expression of protein antigens in their native conformation and that seems to give a higher quality antibody response, which may be one reason for their mysterious priming ability. He also says that understanding how antigens are expressed and processed by cells is key. "A DNA vaccine is not magic. It is a tool to help you modify and optimize antigens in combination-that's the powerful part of DNA vaccines. Everyone who understands that will move forward," he says.

The popularity of the DNA prime- and viral vector-boost combo may be the result of "the respective failings of each on their own," says Maxygen's Whalen. Whereas DNA vaccines have failed to bring about long-lasting responses, viral vector vaccines cannot be given in sequential doses since patients will develop immunity to the vector itself. Bringing viral vectors into the equation also adds some safety concerns and combination trials increase cost, time and approval paperwork. But companies are betting that increased immunogenicity will trump any added headaches - of the ten ongoing clinical trials of gene-based HIV vaccines, four use combination strategies (see Table 1), as do five of seven DNA vaccine trials planned for next year.

\section{Down the homestretch}

The stability and easy characterization of DNA gives it an advantage over viruses in making vaccines for infectious disease. DNA vaccines offer an alternative that will most likely not require refrigeration - a boon to vaccination in the developing world. But perhaps the most impressive benefit of working with DNA is a quick turnaround time for emerging

\section{Table 1 DNA vaccines in clinical development alone or in combination}

\begin{tabular}{lll} 
Sponsor & Type of DNA vaccine & Phase \\
\hline HIV & & \\
\hline NIH Vaccine Research Center & Naked plasmid & 1 ongoinga \\
Emory University & Prime-boost & 1 ongoing \\
Epimmune & Naked plasmid & 1 ongoing \\
Wyeth-Lederle & Naked plasmid & 1 completed \\
Wyeth-Lederle & Naked plasmid & 1 ongoing \\
UMMS & Prime-boost & 1 ongoing \\
Chiron & Prime-boost & 1 ongoing \\
University of New South Wales & Prime-boost & 1 \& \\
& & \\
Cancer & & 1 ongoing \\
\hline ImClone Systems/ & Naked plasmid & 1 completed \\
Memorial Sloan-Kettering Cancer Center & Naked plasmid & 1 \& \\
CTL ImmunoTherapies & Naked plasmid & 2 completed \\
CTL ImmunoTherapies & Naked plasmid & 1 \& \\
National Cancer Institute & Naked plasmid & 1 ongoing \\
M.D. Anderson Cancer Center & Prime-boost & 2 completed \\
Corixa & Naked plasmid & \\
Vical & & \\
\hline Ebola & & 1 ongoing \\
\hline NIH Vaccine Research Center & Naked plasmid & \\
\hline aTwo trials. Source: http://clinicaltrials.gov/ & & \\
& & \\
\hline
\end{tabular}

epidemics. In the outbreaks of both West Nile in North America and SARS (severe acquired respiratory syndrome) in China, the DNA constructs for use in clinical testing were available in just 4-6 months as possible antigen gene sequences became known. Clinical grade traditional vaccines take $2-3$ years to safely develop.

And DNA's safety record is almost perfect in trials so far. DNA injections do not elicit immune reactions to the DNA itself (an early concern in the field) and usually evoke no other adverse reactions. Another safety concern has been the possibility of integration of the DNA plasmid into the patient's chromosomal DNA, which might lead to cancer or other disease-causing mutations. Although human tests for this risk are nearly impossible, extensive animal tests by Vical showed that integration occurs at a rate that is about $1 / 3000$ the rate of spontaneous mutations 4 .

In fact, it was the safety profile of DNA vaccines that convinced Los Angeles Zoo veterinarian Cynthia Stringfield to use them experimentally to protect endangered California condors from West Nile virus (Nat. Biotechnol. 21, 11, 2003). "If there was any risk at all, we probably wouldn't have done it, but there are only 200 of these birds and they are sitting ducks for this disease," she says. (Although the birds have yet to be exposed to the virus, Stringfield says they still show high titers of antibodies almost one year after vaccination.) Despite the excellent safety record, $\mathrm{Ng}$ says the USDA has come back to Fort Dodge requesting additional data, which signifies regulators' caution with the new technology.

\section{Into the winner's circle?}

If Fort Dodge's West Nile virus vaccine were to receive approval, an already energized area could gain even more momentum and a human vaccine could be approved within the next few years. The technology's ability to protect primates exposed to Ebola ${ }^{5}$ and $\mathrm{HIV}^{6}$, and horses from West Nile virus ${ }^{7}$ indicates DNA vaccines hold promise for protecting humans from some of our most ancient (malaria), tricky (HIV) and newly emerging (SARS) infectious enemies. As Harriet Robinson, a DNA vaccine veteran and chief of microbiology and immunology at the Emory University Vaccine Center in Atlanta, puts it: "It's really quite exciting to have a DNA vaccine approval within 12 years of people laughing at the first reports of it."

Kendall Powell is a freelance writer based in Broomfield, Colorado.

1. Wolff, J. et al. Science 247, 1465-1468 (1990).

2. Jiao, S. et al. Hum. Gen. Ther. 3, 21-33 (1992).

3. Whalen, R. Emerg. Infect. Dis. 2, 168-175 (1996).

4. Martin, T. et al. Hum. Gene Ther. 10, 759-768 (1999).

5. Sullivan, N.J. et al. Nature 408, 605-609 (2000).

6. Amara, R.R. et al. Science 292, 69-74 (2001).

7. Davis, B.S. et al. J. Virol. 75, 4040-4047 (2001). 